\title{
BEST PROXIMITY POINT THEOREMS FOR MULTI-VALUED MAPPINGS IN COMPLETE METRIC SPACES
}

\author{
M. R. YADAV, A. K. SHARMA AND B. S. THAKUR
}

\begin{abstract}
In this paper the concept of $\mathrm{K}$-cyclic and $\mathrm{C}$-cyclic contraction single-valued maps are extended to multi-valued maps with MTfunctions in the frameworks of complete spaces. We show the existence of a best proximity point for such mappings in the setup of complete metric spaces. Our result extends and improves some best proximity point theorems in the literature. An example is given to support the functionality of the result.
\end{abstract}

\section{INTRODUCTION:}

In 1969, Nadler [12] studied fixed points of contraction multi-valued mappings. Let $(X, d)$ be a metric space and let $P(X)$ denote the family of all nonempty subsets of $X$. A mapping $T: X \rightarrow P(X)$ is called a multi-valued function. If there exists a constant $k \in(0,1)$ such that $H(T x, T y) \leq k d(x, y)$, for all $x, y \in X$, then we say that $\mathrm{T}$ is a multi-valued contraction mapping. Note that $H(A, B)=\max \{h(A, B), h(B, A)\}$, where $h(A, B)=\sup \{d(a, B): a \in A\}$.

It is also shown in [20] that a mapping $\mathrm{T}$ of $\mathrm{X}$ into the family $\mathrm{K}(\mathrm{X})$ of all nonempty compact subsets of $\mathrm{X}$ has a fixed point if it satisfies $H(T x, T y) \leq$ $k(d(x, y)) d(x, y)$ for all $x, y \in X$ with $x \neq y$, where $k$ is a function of $(0, \infty)$ to $[0,1)$ with $\limsup \operatorname{sut}_{r \rightarrow t+} k(r)<1$ for every $t \in(0, \infty)$.

Let $\mathrm{A}$ and $\mathrm{B}$ be nonempty subsets of a metric space $(X, d)$. Consider a mapping $T: A \cup B \rightarrow A \cup B, T$ is called a cyclic map if $T(A) \subset B$ and $T(B) \subset A, x \in A$ is called a best proximity point of $T$ in $A$ if $d(x, T x)=$ $d(A, B)$ is satisfied, where $d(A, B)=\inf \{d(x, y): x \in A, y \in B\}$.

In a recent paper, Erdal Karapinar and Inci Erhan [4] studied some proximity points by using different types cyclic contraction. Furthermore

2010 Mathematics Subject Classification. 41A65, 46B20, 47H10, 46J10, 46J15.

Key words and phrases. Best proximity point, multivalued mappings, cyclic contraction and complete metric space. 
$[15,5,7]$ examine several variants of contractions for the existence of a best proximity point. Later, Karapinar, E. [6] have derived a best proximity point theorem for Cyclic Mappings. In 2005, Elderd et al. [2] proved the existence of a best proximity point for relatively nonexpansive mappings using the notion of proximal normal structure. In 2006, Eldred and Veeramani [3] proved the following existence theorem. Recently, best proximity point theorems for various types of contractions have been obtained in $[1,8,9,10,14,16]$.

Definition 1.1. Let $A$ and $B$ be nonempty subsets of a metric space $(X, d)$. The cyclic (on $A$ and $B$ ) multivalued mapping $\mathrm{T}$ is said to be cyclic contraction if there exists a constant $k \in(0,1)$ such that $H(T x, T y) \leq$ $k d(x, y)+(1-k) d(A, B)$ for all $x \in A$ and $y \in B$.

Theorem 1.2. Let $A$ and $B$ be nonempty closed convex subsets of a uniformly convex Banach space. Suppose $f: A \cup B \rightarrow A \cup B$ is a cyclic contraction, that is, $f(A) \subset B$ and $f(B) \subset A$, and there exists $k \in(0,1)$ such that $d(f x, f y) \leq k d(x, y)+(1-k) d(A, B)$ for every $x \in A, y \in B$.

Then there exists a unique best proximity point in $A$. Further, for each $x \in A,\left\{f^{2 n} x\right\}$ converges to the best proximity point.

Remark. The following properties of the functional $\mathrm{H}$ are well-known:

(1) $H$ is a metric on $C B(X)$, where $C B(X)$ is the family of all nonempty bounded closed subsets of $X$.

(2) $f(X, d)$ is a metric space, $A, B \in P(X)$ and $q>1$ be given, then for every $a \in A$ there exists $b \in B$ such that $d(a, b) \leq q H(A, B)$.

An element $x \in X$ is a fixed point of a multi-valued $T$ if $x \in T x$. We denote by $F_{T}$ the set of all fixed points of $T$, i.e., $F_{T}=\{x \in X: x \in T x\}$. Theorem 1.3 is a result of [12].

Theorem 1.3. Let $(X, d)$ be a metric space and $T: X \rightarrow C B(X)$ be a multi-valued contraction. Then $T$ has at least one fixed point.

\section{Preliminaries}

In this section, we first define in what follows the MT-function which will be used throughout the paper to get new best proximity point theorems.

Definition 2.1. (See [23]) A function $\psi:[0, \infty) \rightarrow[0,1)$ is said to be an MT-function if it satisfies Mizoguchi-Takahashi's condition $\left(\lim _{s \rightarrow t^{+}} \psi(s)<\right.$ 1 for all $t \in[0, \infty))$.

It is obvious that if $\psi:[0, \infty) \rightarrow[0,1)$ is a non-decreasing function or a non-increasing function, then $\psi$ is an MT-function. So, the set of MTfunctions is a rich class, but it is worth to mention that there exist functions which are not MT-function. 
Example 2.2. (See [25]) Let $\psi:[0, \infty) \rightarrow[0,1)$ be defined by

$$
\psi(t)= \begin{cases}\frac{\sin t}{t} & t \in\left(0, \frac{\pi}{2}\right], \\ 0 & \text { otherwise }\end{cases}
$$

Since $\lim _{t \rightarrow 0^{+}} \psi(s)=1, \psi$ is not an MT-function.

Very recently, Du [25] first proved some characterizations of MT-functions.

Theorem 2.3. Let $\psi:[0, \infty) \rightarrow[0,1)$ be a function. Then the following statements are equivalent.

(a) psi is an MT-function,

(b) For each $t \in[0, \infty)$, there exists $r_{t}^{(1)}$ and $\varepsilon_{t}^{(1)}>0$ such that $\psi(s)<r_{t}^{(1)}$ for all $s \in\left(t, t+\varepsilon_{t}^{(1)}\right)$,

(c) For each $t \in[0, \infty)$, there exists $r_{t}^{(2)}$ and $\varepsilon_{t}^{(2)}>0$ such that $\psi(s)<r_{t}^{(2)}$ for all $s \in\left(t, t+\varepsilon_{t}^{(2)}\right)$,

(d) For each $t \in[0, \infty)$, there exists $r_{t}^{(3)}$ and $\varepsilon_{t}^{(3)}>0$ such that $\psi(s)<r_{t}^{(3)}$ for all $s \in\left(t, t+\varepsilon_{t}^{(3)}\right)$,

(e) For each $t \in[0, \infty)$, there exists $r_{t}^{(4)}$ and $\varepsilon_{t}^{(4)}>0$ such that $\psi(s)<r_{t}^{(4)}$ for all $s \in\left(t, t+\varepsilon_{t}^{(4)}\right)$,

(f) For any non-increasing sequence $\left\{x_{n}\right\}_{n \in N}$ in $[0, \infty)$, we have $0 \leq \sup _{n \in N}$ $\psi\left(x_{n}\right)<L$,

(g) $\psi$ is a function of contractive factor [24]; that is, for any strictly decreasing sequence $\left\{x_{n}\right\}_{n \in N}$ in $[0, \infty)$, we have $0 \leq \sup _{n \in N} \psi\left(x_{n}\right)<1$.

Definition 2.4. [26] Let $A$ and $B$ be nonempty subsets of a metric space $(X, d)$. If a map $T: A \cup B \rightarrow A \cup B$ satisfies:

(a) $T(A) \subset B$ and $T(B) \subset A$;

(b) there exists an MT-function $\psi:[0, \infty) \rightarrow[0,1)$ such that

$$
d(T x, T y) \leq \psi(d(x, y)) d(x, y)+(1-\psi(d(x, y))) d(A, B),
$$

for all $x \in A$ and $y \in B$. Then $T$ is called an MT-cyclic contraction with respect to $\psi$ on $A \cap B$.

Motivated by the definition of $\mathrm{K}$-cyclic, $\mathrm{C}$-cyclic and MT-functions, we introduce the following concept of generalized cyclic contractions.

Definition 2.5. Let $A$ and $B$ be nonempty subsets of a metric space $(X, d)$. If a mapping $T: A \cup B \rightarrow C l(A) \cup C l(B)$ is a generalized cyclic MT-KC contractive map if it is satisfies the following conditions:

(a) $T(A) \subset C l(B)$ and $T(B) \subset C l(A)$;

(b) There exists an MT-function $\psi:[0, \infty) \rightarrow[0,1)$ such that 


$$
\begin{aligned}
H(T x, T y) \leq & \frac{1}{4} \psi(d(x, y))[d(x, T x)+d(x, T y)+d(y, T x)+d(y, T y) \\
& +(1-\psi(d(x, y))) d(A, B)
\end{aligned}
$$

for all $x \in A$ and $y \in B$.

\section{MAIN RESUltS}

In this section, we shall prove the existence and uniqueness convergence theorems for multi-valued mappings to a best proximity points of the nonself-mappings $A$ and $B$.

Theorem 3.1. Let $(A, B)$ be a pair of two nonempty closed subsets of a complete metric space $(X, d)$. Suppose that a mapping $T: A \cup B \rightarrow C l(A) \cup$ $C l(B)$ be a generalized cyclic $M T-K C$ contractive map, then there exits an orbit $\left\{x_{n}\right\}$ of $T$ at $x_{0}$ such that

$$
\lim _{n \rightarrow \infty} d\left(x_{n}, x_{n+1}\right)=d(A, B) .
$$

Proof. Let $x_{0} \in A$ and $x_{1} \in T x_{0} \subset B$. There exists $x_{2} \in T x_{1} \subset A$ such that

$$
\begin{aligned}
& d\left(x_{1}, x_{2}\right) \leq d\left(x_{1}, T x_{1}\right) \leq h\left(T x_{0}, T x_{1}\right) \leq H\left(T x_{0}, T x_{1}\right) \\
& \leq \frac{1}{4} \psi\left(d\left(x_{0}, x_{1}\right)\right)\left[d\left(x_{0}, T x_{0}\right)+d\left(x_{0}, T x_{1}\right)+d\left(x_{1}, T x_{0}\right)+d\left(x_{1}, T x_{1}\right)\right] \\
&+\left(1-\psi\left(d\left(x_{0}, x_{1}\right)\right)\right) d(A, B), \\
& \leq \frac{1}{4} \psi\left(d\left(x_{0}, x_{1}\right)\right)\left[d\left(x_{0}, x_{1}\right)+d\left(x_{0}, x_{2}\right)+d\left(x_{1}, x_{1}\right)+d\left(x_{1}, x_{2}\right)\right] \\
&+\left(1-\psi\left(d\left(x_{0}, x_{1}\right)\right)\right) d(A, B), \\
& \leq \frac{1}{4} \psi\left(d\left(x_{0}, x_{1}\right)\right)\left[2 d\left(x_{0}, x_{1}\right)+2 d\left(x_{1}, x_{2}\right)\right]+\left(1-\psi\left(d\left(x_{0}, x_{1}\right)\right)\right) d(A, B)
\end{aligned}
$$

which implies that

$$
\begin{gathered}
{\left[1-\frac{1}{2} \psi\left(d\left(x_{0}, x_{1}\right)\right)\right] d\left(x_{1}, x_{2}\right) \leq \frac{1}{2} \psi\left(d\left(x_{0}, x_{1}\right)\right) d\left(x_{0}, x_{1}\right)} \\
+\left(1-\psi\left(d\left(x_{0}, x_{1}\right)\right)\left(d\left(x_{0}, x_{1}\right)\right)\right) d(A, B) \\
d\left(x_{1}, x_{2}\right) \leq \frac{\psi\left(d\left(x_{0}, x_{1}\right)\right)}{2-\psi\left(d\left(x_{0}, x_{1}\right)\right)} d\left(x_{0}, x_{1}\right)+\left[1-\frac{\psi\left(d\left(x_{0}, x_{1}\right)\right)}{2-\psi\left(d\left(x_{0}, x_{1}\right)\right)} d(A, B)\right] .
\end{gathered}
$$

From (3.1), finally we obtain

$$
d\left(x_{1}, x_{2}\right)-d(A, B) \leq \frac{\psi\left(d\left(x_{0}, x_{1}\right)\right)}{2-\psi\left(d\left(x_{0}, x_{1}\right)\right)}\left[d\left(x_{0}, x_{1}\right)-d(A, B)\right]
$$


Similarly, there exists $x_{3} \in T x_{2} \subseteq B$ such that

$$
\begin{aligned}
& d\left(x_{2}, x_{3}\right)=H\left(T x_{1}, T x_{2}\right)+h=H\left(T x_{1}, T x_{2}\right) \\
& \leq \frac{1}{4} \psi\left(d\left(x_{1}, x_{2}\right)\right)\left[d\left(x_{1}, T x_{1}\right)+d\left(x_{1}, T x_{2}\right)+d\left(x_{2}, T x_{1}\right)+d\left(x_{2}, T x_{2}\right)\right] \\
&+\left(1-\psi\left(d\left(x_{1}, x_{2}\right)\right)\right) d(A, B), \\
& \leq \frac{1}{4} \psi\left(d\left(x_{1}, x_{2}\right)\right)\left[d\left(x_{1}, x_{2}\right)+d\left(x_{1}, x_{3}\right)+d\left(x_{2}, x_{2}\right)+d\left(x_{2}, x_{3}\right)\right] \\
& \quad+\left(1-\psi\left(d\left(x_{1}, x_{2}\right)\right)\right) d(A, B), \\
& \leq \frac{1}{4} \psi\left(d\left(x_{1}, x_{2}\right)\right)\left[2 d\left(x_{1}, x_{2}\right)+2 d\left(x_{2}, x_{3}\right)\right]+\left(1-\psi\left(d\left(x_{1}, x_{2}\right)\right)\right) d(A, B)
\end{aligned}
$$

which implies that

$$
\begin{aligned}
& {\left[1-\frac{1}{2} \psi\left(d\left(x_{1}, x_{2}\right)\right)\right] d\left(x_{2}, x_{3}\right)} \\
& \leq \frac{1}{2} \psi\left(d\left(x_{1}, x_{2}\right)\right) d\left(x_{1}, x_{2}\right)+\left(1-\psi\left(d\left(x_{1}, x_{2}\right)\right)\right) d(A, B) \\
& d\left(x_{2}, x_{3}\right) \leq \frac{\psi\left(d\left(x_{1}, x_{2}\right)\right)}{2-\psi\left(d\left(x_{1}, x_{2}\right)\right)} d\left(x_{1}, x_{2}\right)+\left[1-\frac{\psi\left(d\left(x_{1}, x_{2}\right)\right)}{2-\psi\left(d\left(x_{1}, x_{2}\right)\right)}\right] d(A, B)
\end{aligned}
$$

or

$$
d\left(x_{2}, x_{3}\right)-d(A, B) \leq \frac{\psi\left(d\left(x_{1}, x_{2}\right)\right)}{2-\psi\left(d\left(x_{1}, x_{2}\right)\right)}\left[d\left(x_{1}, x_{2}\right)-d(A, B)\right]
$$

By induction, we get

$$
d\left(x_{n}, x_{n+1}\right)-d(A, B) \leq \frac{\psi\left(d\left(x_{n-1}, x_{n}\right)\right)}{2-\psi\left(d\left(x_{n-1}, x_{n}\right)\right)}\left[d\left(x_{n-1}, x_{n}\right)-d(A, B)\right] .
$$

Since $\psi(t)<1$ for all $t \in[0, \infty)$, where $\frac{\psi(t)}{1-\psi(t)}<1$ for all $t \in[0, \infty)$. By (3.2), we obtain

$$
d\left(x_{n}, x_{n+1}\right)-d(A, B) \leq d\left(x_{n-1}, x_{n}\right)-d(A, B),
$$

which implies that $d\left(x_{n}, x_{n+1}\right)<d\left(x_{n-1}, x_{n}\right)$ for all $n \in N$. Thus the sequence $\left\{d\left(x_{n}, x_{n+1}\right)\right\}$ is a strictly decreasing in $[0, \infty)$. Since $\psi$ is an MTfunction by applying (g) by Theorem 2.3 , we get

$$
0 \leq \sup _{n \in N} \psi\left(d\left(x_{n}, x_{n+1}\right)\right)<1 .
$$

Suppose $k=\sup _{n \in N} \psi\left(d\left(x_{n}, x_{n+1}\right)\right)$. Then $0 \leq k<1$, since $\psi\left(d\left(x_{n}, x_{n+1}\right)\right)$ $\leq k$, we get

$$
1-\psi\left(d\left(x_{n}, x_{n+1}\right)\right) \geq 1-k
$$

Then

$$
\frac{\psi\left(d\left(x_{n}, x_{n+1}\right)\right)}{1-\psi\left(d\left(x_{n}, x_{n+1}\right)\right)} \leq \frac{k}{1-k}
$$


for all $n \in N$. Hence

$$
0 \leq \sup _{n \in N} \frac{\psi\left(d\left(x_{n}, x_{n+1}\right)\right)}{1-\psi\left(d\left(x_{n}, x_{n+1}\right)\right)} \leq \frac{k}{1-k}<1
$$

Call

$$
\alpha=\sup _{n \in N} \frac{\psi\left(d\left(x_{n}, x_{n+1}\right)\right)}{1-\psi\left(d\left(x_{n}, x_{n+1}\right)\right)} .
$$

Then $\alpha \in[0,1)$. From $(3.2)$, we have

$$
\begin{aligned}
d\left(x_{n}, x_{n+1}\right)-d(A, B) & \leq \frac{\left.\psi\left(d\left(x_{n-1}, x_{n}\right)\right)\right)}{1-\psi\left(d\left(x_{n-1}, x_{n}\right)\right)}\left[d\left(x_{n-1}, x_{n}\right)-d(A, B)\right] \\
& \leq \alpha\left[d\left(x_{n-1}, x_{n}\right)-d(A, B)\right] \\
& \leq \alpha^{2}\left[d\left(x_{n-2}, x_{n-1}\right)-d(A, B)\right] \\
& \cdots \\
& \leq \alpha^{n}\left[d\left(x_{0}, x_{1}\right)-d(A, B)\right] .
\end{aligned}
$$

Since $\alpha \in[0,1)$ and taking $n \rightarrow \infty$ in (3.3), we have $\lim _{n \rightarrow \infty} \alpha^{n}=0$, So that, we obtain

$$
\lim _{n \rightarrow \infty} d\left(x_{n}, x_{n+1}\right)=d(A, B) .
$$

This completes the proof.

Theorem 3.2. Let $(A, B)$ be a pair of two nonempty closed subsets of a complete metric space $(X, d)$. Suppose that a mapping $T: A \cup B \rightarrow C l(A \cup B)$ be a generalized cyclic $M T-K C$ contractive map. Assume that a sequence $\left\{x_{2 n}\right\}$ has a subsequence converging to some element $x$ in $A$. Then the sequence $\left\{x_{n}\right\}$ is bounded.

Proof. Suppose $x_{2 n} \in T x_{2 n-1} \subset B$. Then, there exists $x_{2 n+1} \in T x_{2 n} \subset A$ such that

$$
\begin{aligned}
& d\left(x_{2 n}, T x_{0}\right)=H\left(T x_{2 n-1}, T x_{0}\right) \\
& \leq \frac{1}{4} \psi\left(d\left(x_{2 n-1}, x_{0}\right)\right)\left[d\left(x_{2 n-1}, T x_{2 n-1}\right)+d\left(x_{2 n-1}, T x_{0}\right)\right. \\
&+d\left(x_{0}, T x_{2 n-1}\right)+d\left(x_{0}, T x_{0}\right)+\left(1-\psi\left(d\left(x_{2 n-1}, x_{0}\right)\right)\right) d(A, B) \\
& \leq \frac{1}{4} \psi\left(d\left(x_{2 n-1}, x_{0}\right)\right)\left[d\left(x_{2 n-1}, x_{2 n}\right)+d\left(x_{2 n-1}, T x_{0}\right)\right. \\
&\left.+d\left(x_{0}, x_{2 n}\right)+d\left(x_{0}, T x_{0}\right)\right]+\left(1-\psi\left(d\left(x_{2 n-1}, x_{0}\right)\right)\right) d(A, B) \\
& \leq \frac{1}{4} \psi\left(d\left(x_{2 n-1}, x_{0}\right)\right)\left[d\left(x_{2 n-1}, x_{2 n}\right)+d\left(x_{2 n-1}, x_{2 n}\right)+d\left(x_{2 n}, T x_{0}\right)+d\left(x_{0}, T x_{0}\right)\right. \\
&\left.+d\left(x_{2 n}, T x_{0}\right)+d\left(x_{0}, T x_{0}\right)\right]+\left(1-\psi\left(d\left(x_{2 n-1}, x_{0}\right)\right)\right) d(A, B) \\
& \leq \frac{1}{4} \psi\left(d\left(x_{2 n-1}, x_{0}\right)\right)\left[2 d\left(x_{2 n-1}, x_{2 n}\right)+2 d\left(x_{2 n}, T x_{0}\right)+2 d\left(x_{0}, T x_{0}\right)\right]
\end{aligned}
$$




$$
\begin{aligned}
& +\left(1-\psi\left(d\left(x_{2 n-1}, x_{0}\right)\right)\right) d(A, B) \\
\leq & \frac{1}{2} \psi\left(d\left(x_{2 n-1}, x_{0}\right)\right)\left[d\left(x_{2 n-1}, x_{2 n}\right)+d\left(x_{2 n}, T x_{0}\right)+d\left(x_{0}, T x_{0}\right)\right] \\
& +\left(1-\psi\left(d\left(x_{2 n-1}, x_{0}\right)\right)\right) d(A, B) \\
\leq & \frac{\psi\left(d\left(x_{2 n-1}, x_{0}\right)\right)}{2-\psi\left(d\left(x_{2 n-1}, x_{0}\right)\right)}\left[d\left(x_{2 n-1}, x_{2 n}\right)+d\left(x_{0}, T x_{0}\right)\right] \\
& +\left(1-\frac{\psi\left(d\left(x_{2 n-1}, x_{0}\right)\right)}{2-\psi\left(d\left(x_{2 n-1}, x_{0}\right)\right)}\right) d(A, B) \\
\leq & \frac{\psi\left(d\left(x_{2 n-1}, x_{0}\right)\right)}{2-\psi\left(d\left(x_{2 n-1}, x_{0}\right)\right)}\left[d(A, B)+d\left(x_{0}, T x_{0}\right)\right] \\
& +\left(1-\frac{\psi\left(d\left(x_{2 n-1}, x_{0}\right)\right)}{2-\psi\left(d\left(x_{2 n-1}, x_{0}\right)\right)}\right) d(A, B) \\
\leq & \frac{\psi\left(d\left(x_{2 n-1}, x_{0}\right)\right)}{2-\psi\left(d\left(x_{2 n-1}, x_{0}\right)\right)} d\left(x_{0}, T x_{0}\right)+d(A, B) .
\end{aligned}
$$

Therefore, the sequence $\left\{x_{2 n}\right\}$ is bounded. Similarly, it can be shown that $\left\{x_{2 n+1}\right\}$ is also bounded. This complete the proof.

Theorem 3.3. Let $(A, B)$ be a pair of two nonempty closed subsets of a complete metric space $(X, d)$. Let a mapping $T: C l(A) \cup C l(B) \rightarrow C l(A \cup B)$ be a generalized cyclic $M T-K C$ contractive map. Suppose that a sequence $\left\{x_{2 n}\right\}$ has a subsequence converging to some element $x$ in $A$. Then $x$ is a best proximity point of $T$.

Proof. Suppose the sequence $\left\{x_{2 n_{k}}\right\}$ is a subsequence of $\left\{x_{2 n}\right\}$ converging to some element $x$ in $A$. Furthermore,

$$
\begin{aligned}
d(A, B) & \leq d\left(x, x_{2 n_{k}-1}\right) \\
& \leq d\left(x, x_{2 n_{k}}\right)+d\left(x_{2 n_{k}}, x_{2 n_{k}-1}\right) \\
& \leq d\left(x, x_{2 n_{k}}\right)+d(A, B)
\end{aligned}
$$

Therefore $d\left(x, x_{2 n_{k}-1}\right) \rightarrow d(A, B)$. In light of the fact that the sequence $\left\{x_{2 n_{k}}\right\}$ has a subsequence of $\left\{x_{2 n}\right\}$ converging to some element $x$ in $A$. So, because of Theorem 3.1 e have $d\left(x_{2 n_{k}}, x_{2 n_{k}-1}\right) \rightarrow d(A, B)$. Since $T$ is a generalized cyclic MT-KC contractive map cyclic contraction, it follows that

$$
\begin{aligned}
& d(A, B) \leq d\left(x_{2 n_{k}}, T x\right)=H\left(T x_{2 n_{k}-1}, T x\right) \\
& \leq \frac{1}{4} \psi\left(d\left(x_{2 n_{k}-1}, x\right)\right)\left[d\left(x_{2 n_{k}-1}, T x_{2 n_{k}-1}\right)+d\left(x_{2 n_{k}-1}, T x\right)\right. \\
& \left.\quad+d\left(x, T x_{2 n_{k}-1}\right)+d(x, T x)\right]+\left(1-\psi\left(d\left(x_{2 n_{k}-1}, x\right)\right)\right) d(A, B)
\end{aligned}
$$




$$
\begin{aligned}
\leq & \frac{1}{4} \psi\left(d\left(x_{2 n_{k}-1}, x\right)\right)\left[d\left(x_{2 n_{k}-1}, x_{2 n_{k}}\right)+d\left(x_{2 n_{k}-1}, T x\right)\right. \\
& \left.+d\left(x, x_{2 n_{k}}\right)+d(x, T x)\right]+\left(1-\psi\left(d\left(x_{2 n_{k}-1}, x\right)\right)\right) d(A, B) \\
\leq & \frac{1}{4} \psi\left(d\left(x_{2 n_{k}-1}, x\right)\right)\left[d\left(x_{2 n_{k}-1}, x_{2 n_{k}}\right)+d\left(x_{2 n_{k}-1}, x_{2 n_{k}}\right)+d\left(x_{2 n_{k}}, T x\right)\right. \\
& \left.+d(x, T x)+d\left(x_{2 n_{k}}, T x\right)+d(x, T x)\right]+\left(1-\psi\left(d\left(x_{2 n_{k}-1}, x\right)\right)\right) d(A, B) \\
\leq & \frac{1}{4} \psi\left(d\left(x_{2 n_{k}-1}, x\right)\right)\left[2 d\left(x_{2 n_{k}-1}, x_{2 n_{k}}\right)+2 d\left(x_{2 n_{k}-1}, T x\right)+2 d(x, T x)\right] \\
& +\left(1-\psi\left(d\left(x_{2 n_{k}-1}, x\right)\right)\right) d(A, B) \\
\leq & \frac{1}{2} \psi\left(d\left(x_{2 n_{k}-1}, x\right)\right)\left[d\left(x_{2 n_{k}-1}, x_{2 n_{k}}\right)+d\left(x_{2 n_{k}}, T x\right)+d(x, T x)\right] \\
& +\left(1-\psi\left(d\left(x_{2 n_{k}-1}, x\right)\right)\right) d(A, B) \\
\leq & \frac{\psi\left(d\left(x_{2 n_{k}-1}, x\right)\right)}{2-\psi\left(d\left(x_{2 n_{k}-1}, x\right)\right)}\left[d\left(x_{2 n_{k}-1}, x_{2 n_{k}}\right)+d(x, T x)\right] \\
& +\left(1-\frac{\psi\left(d\left(x_{2 n_{k}-1}, x\right)\right)}{2-\psi\left(d\left(x_{2 n_{k}-1}, x\right)\right)}\right) d(A, B) \\
\leq & \frac{\psi\left(d\left(x_{2 n_{k}-1}, x\right)\right)}{2-\psi\left(d\left(x_{2 n_{k}-1}, x\right)\right)} d(x, T x)+d(A, B) .
\end{aligned}
$$

Since $\psi$ is an MT-function by applying (g) by Theorem 2.3, we get

$$
0 \leq \sup _{n \in N} \psi\left(d\left(x_{n}, x_{2 n_{k}-1}\right)\right)<1 .
$$

and taking $k \rightarrow \infty$ in the inequality above, then we obtain

$$
d(x, T x)=d(A, B),
$$

that is $x$ is a best proximity point of $T$. This completes the proof.

The following examples illustrate our main results.

Example 3.4. Consider the usual metric space $d(x, y)=|x-y|$, for all $x, y \in X$. Let $X=R$. Suppose $A=[0,1]$ and $B=[2,3]$, then $d(A, B)=1$. Define a mapping $T: A \cup B \rightarrow C l(A) \cup C l(B)$ as follows:

$$
T x=\left[1, \frac{5-x}{2}\right] \text { for all } x \in A \text { s.t. } T x=\{[1, a]: a \in[2,2.5]\}
$$

and

$$
T y=\left[0, \frac{4-y}{2}\right] \text { for all } y \in B \text { s.t. } T y=\{[0, b]: b \in[0.5,1]\} .
$$

It is clear that $T(A) \subset C l(B)$ and $T(B) \subset C l(A)$. Since,

$$
H(T x, T y)=\max \left[\left\{\sup _{x \in A} d(x, T y)\right\},\left\{\sup _{y \in B} d(y, T x)\right\}\right] .
$$


Let us observe that, for $x=1$ and $y=2$, then, we have $T x=[1,2]$ and $T y=[0,1]$. Then, we obtain,

$$
\begin{aligned}
H(T x, T y) & =\max \{[\sup (d(1,0), d(1,1))],[\sup (d(2,1), d(2,2))]\} \\
& =\max \{\sup [1,0], \sup [1,0]\} \\
& =\max \{0,0\}=0 .
\end{aligned}
$$

Similarly, we find that

$$
\begin{aligned}
& d(x, T x)=\sup [d(x, a): a \in T x]=\sup [0,1]=1, \\
& d(y, T y)=\sup [d(y, b): b \in T y]=\sup [2,1]=2, \\
& d(x, T y)=\sup [d(x, b): b \in T y]=\sup [1,0]=1, \\
& d(y, T x)=\sup [d(y, a): a \in T x]=\sup [1,0]=1 .
\end{aligned}
$$

Let $\psi:[0, \infty) \rightarrow[0,1)$ and the constant function given by $\psi(t)=\frac{t}{2(1+t)}$. Therefore, it is easy to check that

$$
\begin{aligned}
H(T x, T y) \leq & \frac{1}{4} \psi(d(x, y))[d(x, T x)+d(x, T y)+d(y, T x)+d(y, T y)] \\
& +(1-\psi(d(x, y))) d(A, B) \\
\leq & \frac{1}{4} \cdot \frac{1}{4}[1+2+1+1]+\left(1-\frac{1}{4}\right) 1=\frac{11}{8} .
\end{aligned}
$$

It is clear that $0 \leq \frac{11}{8}$. Therefore, condition (2.2) is satisfied. So that a mapping $\mathrm{T}$ is a generalized cyclic $\mathrm{MT}-\mathrm{KC}$ contractive map.

\section{Application}

In this section, we present some applications of the main results.

Corollary 4.1. Let $(A, B)$ be a pair of two nonempty closed subsets of a complete metric space $(X, d)$. Suppose that a mapping $T: A \cup B \rightarrow C l(A) \cup$ $\mathrm{Cl}(B)$ satisfying the following condition:

(a) $T(A) \subset C l(B)$ and $T(B) \subset C l(A)$;

(b) there exists an MT-function $\psi:[0, \infty) \rightarrow[0,1)$ such that

$$
\begin{aligned}
\int_{0}^{H(T x, T y)} \mu(t) d t \leq & \frac{1}{4}(1-\psi(d(x, y))) \int_{0}^{[d(x, T x)+d(x, T y)+d(y, T x)+d(y, T y)]} \mu(t) d t \\
& +(1-\psi(d(x, y))) \int_{0}^{d(A, B))} \mu(t) d t,
\end{aligned}
$$

for each $x \in A$ and $y \in B$, where $\mu: R^{+} \rightarrow R^{+}$is a Lesbesgue-integrable mapping which is summable on each compact subset of $R^{+}$, non negative, and such that for each $\epsilon>0, \int_{0}^{\epsilon} \mu(t) d t<\epsilon$. Then, there exists $\lim _{n \rightarrow \infty} d\left(x_{n}, x_{n+1}\right)$ $=d(A, B)$ for some sequence $\left\{x_{n}\right\}$. 
Corollary 4.2. Let $(A, B)$ be a pair of two nonempty closed subsets of a complete metric space $(X, d)$. Suppose that a mapping $T: A \cup B \rightarrow C l(A) \cup$ $C l(B)$ satisfying the following conditions:

(a) $T(A) \subset C l(B)$ and $T(B) \subset C l(A)$;

(b) There exists an $M T$-function $\psi:[0, \infty) \rightarrow[0,1)$ such that

$$
\begin{aligned}
\int_{0}^{H(T x, T y)} \mu(t) d t \leq & \frac{1}{4}(1-\psi(d(x, y))) \int_{0}^{[d(x, T x)+d(x, T y)+d(y, T x)+d(y, T y)]} \mu(t) d t \\
& +(1-\psi(d(x, y))) \int_{0}^{d(A, B))} \mu(t) d t,
\end{aligned}
$$

for each $x \in A$ and $y \in B$, where $\mu: R^{+} \rightarrow R^{+}$is a Lesbesgue-integrable mapping which is summable on each compact subset of $R^{+}$, non negative, and such that for each $\epsilon>0, \int_{0}^{\epsilon} \mu(t) d t<0$. Suppose that a sequence $\left\{x_{2 n}\right\}$ has a subsequence converging to some element $x$ in $A$. Then, there exists $x \in A$ such that $d(x, T x)=d(A, B)$.

Acknowledgments. The authors would like to express their sincere thanks to the referees for their valuable comments on this article and Prof. Harry I. Miller for his helpful remarks, suggestions that allowed them to improve the original paper.

\section{REFERENCES}

[1] A. Almeida, E. Karapinar and K. Sadarangani, A note on best proximity point theorems under weak P-property, Abstr. Appl. Anal., 2014, Article Id: 716825

[2] A. A. Eldred, W. A. Kirk and P. Veeramani, Proximal normal structure and relatively nonexpanisve mappings, Stud. Math., 171 (3) (2005), 283-293.

[3] A. A. Eldred and P. Veeramani, Existence and convergence of best proximity points, J. Math. Anal. Appl., 323 (2006), 1001-1006.

[4] E. Karapinar and I. M. Erhan, Best proximity point on different type contractions, Appl. Math. Inf. Sci., 5 (2011), 342-353.

[5] E. Karapinar, G. Petrusel and K. Tas, Best proximity point theorems for KT-types cyclic orbital contraction mappings, Fixed Point Theory, 13 (2) (2012), 537-546.

[6] E. Karapinar, Best proximity points of cyclic mappings, Appl. Math. Lett., 25 (11) (2012), 1761-1766.

[7] E. Karapinar, On best proximity point of psi-Geraghty contractions, Fixed Point Theory Appl., 2013, 2013:200.

[8] E. Karapinar, V. Pragadeeswarar and M. Marudai, Best proximity point for generalized proximal weak contractions in complete metric space, J. Appl. Math., (2014) Article No: 150941.

[9] E. Karapinar and B. Samet, A note on psi-Geraghty type contractions, Fixed Point Theory Appl., 2014, 2014:26.

[10] H. Aydi, E. Karapinar, I. M. Erhan and P. Salimi, Best proximity points of generalized almost- Geraghty contractive non-self mappings, Fixed Point Theory Appl., 2014, 2014:32. 
[11] H. K. Pathak, Y. J. Cho and S. M. Kang, Remarks on R-weakly commuting mappings and common fixed point theorems, Bull. Korean Math. Soc., 34 (1997), 247-257.

[12] JR. S. B. Nadler, Multi-valued contraction mappings, Pacific J. Math., 30 (1969), 475-488.

[13] J. J. Nieto and R. Rodrguez-Lpez, Contractive mapping theorems in partially ordered sets and applications to ordinary differential equations, Order, 22 (2005), 223-239.

[14] M. De La Sen, E. Karapinar, On best proximity points of generalized semi-cyclic impulsive self-mappings. Applications to impulsive differential and difference equations, Abstr. Appl. Anal., (2013) Article Id: 505487.

[15] M. Jleli, E. Karapinar and B. Samet, On best proximity points under the P-property on partially ordered metric spaces, Abstr. Appl. Anal., 2013 Article Id: 150970.

[16] N. Bilgili, E. Karapinar and K. Sadarangani, A generalization for the best proximity point of Geraghty-contractions, J. Inequal. Appl., (2013), 2013:286.

[17] R. Kannan, Some results on fixed points -II, Bull. Calcutta Math. Soc., 60 (1969), $71-76$.

[18] R. P. Agarwal, M. A. EL-Gebeily and D. ORegan, Generalized contractions in partially ordered metric spaces, Appl. Anal., 87 (2008), 109-116.

[19] S. K. Chatterjee, Fixed point theorems, Com. Ren. Acad. Bulgaria Sci., 25 (1972), 727-730.

[20] S. Reich, Fixed points of contractive functions, Boll. (in. Mar. Ital., 5 (1972), 26-42.

[21] S. Reich, Some fixed point problems, Atri. Acad. Nuz. Lincei, 57 (1974). 194-198.

[22] S. Sessa and Y. J. Cho, Compatible mappings and a common fixed point theorem of Chang type, Publ. Math. (Debrecen), 43 (1993), 289-296.

[23] W. S. Du, Some new results and generalizations in metric fixed point theory, Nonlinear Anal., Theory Methods Appl., 73 (2010), 1439-1446.

[24] W. S. Du, Nonlinear contractive conditions for coupled cone fixed point theorems, Fixed Point Theory Appl. 2010, Article ID 190606 (2010). doi:10.1155/2010/190606.

[25] W. S. Du, On coincidence point and fixed point theorems for nonlinear multivalued maps, Topol. Appl., 159 (2012), 49-56.

[26] W. S. Du and H. Lakzian, Nonlinear conditions for the existence of best proximity points, J. Inequal. Appl., 2012, 2012:206.

[27] W. Sintunavarat, Y. J. Cho and P. Kumam, Common fixed point theorems for cdistance in ordered cone metric spaces, Comput. Math. Appl., 62 (2011) 1969-1978.

[28] Y. J. Cho, Fixed points for compatible mappings of type (A), Math. Jap., 18 (1993), $497-508$.

(Received: November 12, 2013)

(Revised: February 21, 2014)
M.R. Yadav and B.S. Thakur

School of Studies in Mathematics

Pt. Ravishankar Shukla University

Raipur, Chhattisgarh (India)-492010

yadavmryadav@gmail.com

balwantst@gmail.com

\author{
A.K. Sharma \\ Department of Mathematics \\ Seth Phoolchand College, Navapara \\ Rajim, Dist. Raipur, Chhattisgarh (India) \\ sharmakajay17@gmail.com
}

